\title{
Vibrotactile Cueing using Wearable Computers for Overcoming Learned Non-Use in Chronic Stroke
}

\author{
Eric L. Luster ${ }^{1}$, Troy McDaniel ${ }^{1}$, Bijan Fakhri ${ }^{1}$, Jim Davis ${ }^{2}$, \\ Morris Goldberg ${ }^{1}$, Shantanu Bala ${ }^{1}$, and Sethuraman Panchanathan ${ }^{1}$
}

\author{
Center for Cognitive Ubiquitous Computing ${ }^{1}$ \\ Arizona State University, Tempe, Arizona, USA \\ \{eluster, troy.mcdaniel, bijan.fakhri, shantanu.bala, \\ panch\}@asu.edu,mgoldberg@ieee.org
}

\author{
Rehab Arizona ${ }^{2}$ \\ Mesa, Arizona, USA \\ jim.davis@ rehabarizona.com
}

\begin{abstract}
Outpatient stroke rehabilitation is often lengthy and expensive due to patients' lack of functional use of the impaired arm outside of the clinic caused by "learned non-use." Learned non-use is detrimental to stroke recovery, often resulting in chronic disability. To overcome learned non-use, a wearable "personal assistant" solution is proposed that employs ubiquitous cueing to stimulate patient use of the paretic arm while outside of therapy sessions. A pilot user study is presented that evaluated stroke survivors' tolerance and acceptance of cueing, and the usability of the proposed implementation.
\end{abstract}

Keywords-non-use; stroke rehabilitation; vibrotactile cueing.

\section{INTRODUCTION}

About 795,000 Americans experience a stroke every year [1]. Stroke is the leading cause of long-term disability in the United States [1] in which survivors experience partial or complete paralysis on one side of their body. The National Institute of Neurological Disorders and Stroke estimates the total annual cost of stroke in the United States to be approximately $\$ 43$ billion. Chronic disability, which degrades quality of life and increases healthcare costs due to long-term care and treatment needs, is experienced by the majority of stroke survivors, and is attributed to many factors including non-use of the impaired arm.

Limited functional use of the weakened (paretic) arm and little to no adherence to therapist-prescribed home exercises creates an impediment to stroke recovery since frequent functional use is needed to reorganize neuronal circuits to regain neuromuscular control. A common cause of non-use is overreliance on the unimpaired (or less-impaired) arm for functional tasks, explained by the theory, "learned non-use." Learned non-use [2] begins early after stroke due to conditioning: difficulties and failures associated with using the paretic arm are encountered early after stroke, which are circumvented by relying on and compensating with the unimpaired arm, promoting its use over the impaired limb for functional tasks. For example, using wrist-worn accelerometers, Lang et al. [3] found low activity levels in the paretic arm as early as a few weeks after stroke during inpatient treatment: 3.3 hours per day for the paretic arm, and 6 hours per day for the unimpaired arm; this is in contrast to healthy subjects, who average 8-9 hours per day for either upper extremity.
Constraint-induced movement therapy (CIMT) [2] is a therapeutic treatment for overcoming learned non-use in chronic stroke survivors. CIMT forces use of the impaired arm by restricting the unimpaired arm (using a mitt or sling) for $90 \%$ of waking hours during a period of 2-3 weeks involving daily therapy sessions with adaptive training procedures, or "shaping," for up to 6 hours. Shaping [4] is a training method where an objective behavior is sought by teaching incrementally closer approximations to a target behavior. Shaping helps ease patients into incrementally more difficult movements, positively reinforcing successful attempts toward an objective. The success of CIMT is due to both its adaptive training protocols and ubiquity - the mitt or sling is always worn except during sleep, excretory functions and bathing, which forces use of the paretic arm throughout the day during activities of daily living. Although highly effective at overcoming learned non-use, CIMT is prohibitively expensive due to the amount of therapist supervision involved; and unsafe for patients with motor deficits of the lower extremities due to the risk of falling [2].

While robotics, games, and virtual and mixed reality environments have received significant attention over the last few decades for upper extremity stroke rehabilitation, the majority of approaches thus far have not explored ubiquitous solutions based on the highly effective approach of CIMT in which the wearable aid is always worn and in use. Although clinical studies have shown that virtual reality-based robotmediated therapy is highly effective at recovering functions of the upper extremities for chronic stroke survivors [5], the majority of systems are still prohibitively expensive, and require considerable training and supervision. This paper presents a ubiquitous and pervasive wearable "personal assistant" to augment traditional stroke therapy, and complement, rather than replace, existing rehabilitative technologies using cueing to enhance patient awareness of activity levels and non-adherence toward increased use and functional gains. The results of a pilot user study revealed user acceptance, tolerance and enthusiasm for the proposed approach. The rest of the paper is organized as follows: Section II provides an overview of related work on wearable computers for motor learning and rehabilitation; Sections III and IV present the proposed approach and implementation; in Section $\mathrm{V}$, user study results are presented; and Section VI provides directions for future work. 


\section{RELATED WORK}

Wearable computers, such as the Tactile Interaction for Kinesthetic Learning (TIKL) system [6] and StrokeSleeve [7], have been proposed to augment traditional motor learning and rehabilitation using vibrotactile stimulation on the arm to indicate errors in limb positions. Mobile Music Touch [8] is a wireless vibrotactile glove where stimulation on the back of each finger is synchronized to the keys involved in simple piano music. The system is currently being explored for hand rehabilitation of incomplete Spinal Cord Injury (SCI) patients. In terms of commercial products, Bioness offers electrical stimulation-based orthoses including the L300 for foot drop therapy and the H200 Wireless Hand Rehabilitation System for assisting with hand positioning, grasp and release.

Toward the goal of enhancing stroke survivors' awareness of activity levels to overcome learned non-use, Us'em [9] is a wearable computer that delivers visual feedback of use ratios between the paretic arm and unimpaired arm using a bar chart shown on a small display on a pair of wrist-worn devices with embedded accelerometers for motion sensing. While Us'em provides ubiquitous feedback, the approach does not directly challenge the user to increase the activity level of the paretic arm. As previously discussed, the success of CIMT is due to both its ubiquity and adaptive training protocols where shaping is utilized to alter the difficulty of tasks. Furthermore, the interpretation of bar charts may have high cognitive load for many stroke survivors, and may not be applicable to those with visual and/or cognitive impairments. This type of display may also create distractions when visual attention is drawn away from the present task to observe and interpret visual feedback.

\section{PROPOSED APPROACH}

To overcome the limitations of existing approaches for reducing non-use, and replace or lessen the use of CIMT, we propose a wearable personal assistant, depicted in Fig. 1, using vibrotactile cueing to discreetly and unobtrusively increase awareness of non-use of the paretic arm. The proposed approach is inspired by CIMT's ubiquity without restricting the arm. Additionally, our approach is pervasive in that it is intended to integrate with existing protocols of stroke rehabilitation, and both clinic and home-based technologies. The large variance among stroke survivors in terms of the severity of stroke necessitates a "personal" solution in which therapists can specify exercises, goals, and shaping protocols based on patient assessment and progress. Cueing is used to generate awareness of activity levels to promote paretic arm use during activities of daily living (e.g., dressing, grooming, eating, etc.) and adherence to therapist-prescribed home exercises. During activities of daily living, it is not uncommon for the unimpaired arm to substitute for the paretic arm during unimanual tasks, or overcompensate for the paretic arm during bimanual task; we hypothesize that cueing can increase patient awareness of non-use of the paretic arm by conveying how therapist-defined performance goals (e.g., activity levels of the paretic arm) are being met. Moreover, we hypothesize that cueing can increase adherence to therapist-prescribed exercises for the home setting. Cueing to promote functional use and adherence to exercise consists of three stages: sensing, adaptation and delivery.

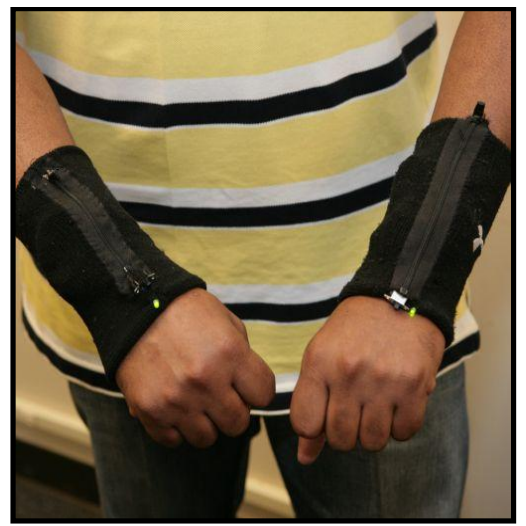

Figure 1. Discreet and unobtrusive vibrotactile cueing using wearable computers embedded in wristbands for enhancing the awareness of nonuse and neglect in hemiparetic stroke survivors.

Sensing involves the use of inertial measurement devices paired with movement detection algorithms for recognizing functional movements of the unimpaired and impaired limbs, and the estimation of activity levels. In the proposed implementation, accelerometry is used for motion sensing as it has been shown to provide objective, continuous data capture of the intensity of physical activity [10]. To detect functional movements of the upper extremities, Uswatte et al.'s threshold filter approach [10] was used (see Section IV), which has been shown to accurately measure the duration of upper extremity movements of stroke survivors.

Adaptation involves the use of computational methods for dynamically updating performance goals (in this case, desired activity levels) where both the goal itself and shaping procedure may be initially set (and continually adjusted) by the therapist based on a stroke survivor's clinical assessment, outcome measures and observed progress throughout intervention. Activity levels (e.g., "the impaired arm should be used $40 \%$ of waking hours") are dynamically adapted (shaped) toward an objective goal (e.g., "70\% of waking hours"). Adaptation may involve increasing or decreasing the required level of activity of the paretic arm based on the performance of the user. In this situation, shaping may be employed to support incremental sub-goals toward an overall objective, helping to reduce frustration while positively reinforcing small successes.

Cueing may act as a reminder or prompt, but also, provide positive reinforcement when performance goals are met; or awareness of unsatisfactory performance when goals are not met. The delivery modality (visual, auditory, vibrotactile, etc.) is important to consider, as well as stimulation designs that are hard-to-miss and intuitive to learn. For the proposed implementation, vibrotactile cues were chosen for their inherent discreetness; moreover, they do not require visual attention. However, for patients with reduced tactile sensitivity, additional auditory or multimodal cues may be preferred.

\section{IMPLEMENTATION}

Each wristband consists of a microcontroller (LilyPad Arduino 328 Main Board); triple axis accelerometer (LilyPad Accelerometer ADXL335) for motion sensing; pancake motor (Precision Microdrives, model\#: 312-101) for vibrotactile 
stimulation; MicroSD card breakout board for accelerometer data storage and retrieval; Zigbee protocol-based XBee Series 1 module for (optional) wireless transmission of accelerometer data; rechargeable battery for power; on/off switch; and power indicator (LED). The electronics are embedded within an Under Armour 6-inch wristband modified with a zipper to easily insert and access the electronics.

Acceleration is sampled at $10 \mathrm{~Hz}$, and for each sample, the readings for the $x, y$ and $z$ axes are used to form a force vector with magnitude $M$. The threshold filter approach [10] is used to measure the duration of upper extremity movement. In the original approach, bandpass filtered accelerations are sampled at $10 \mathrm{~Hz}$ over a 2 -second period (epoch), and then summed to create a raw count, which is then compared to a threshold; if the raw count is above the threshold, then functional movement occurred within the epoch. Epochs in which valid movements occurred are summed to obtain an estimate of the duration of movement. For the proposed implementation, some minor adjustments to the threshold filter approach were made based on our model of accelerometer. The main difference is the use of a larger threshold value, $1.1 \mathrm{~g}$, to detect functional movement (where at least $1 \mathrm{~g}$ accounts for gravity) due to not using a bandpass filter. Moreover, two such samples are required during a single epoch for robustness. Pilot tests revealed accurate detection of intentional movements such as reaching and grasping. Use ratios are calculated as the sum of epochs in which movement occurred to the sum of all epochs.

\section{PILOT USER STUDY}

Aim: Before evaluating the effectiveness of cueing for lessening non-use and promoting adherence to exercises for stroke survivors, a preliminary IRB-approved user study was conducted to explore user tolerance and acceptance of vibrotactile cueing, and receive feedback on usability.

Subjects: Five stroke survivors (2 males, 3 females) completed the study. One participant was between the ages of 22-34; two were between the ages of 45-54; and two were between the ages of 55-64. For four of the five participants, the more-impaired arm was the right arm, and for all participants, the right arm was dominant. All participants suffered no more than one stroke with the average number of years since the stroke being 1.21 (SD: 0.51). Participant screening and recruitment occurred through a local rehabilitation clinic under the supervision of a licensed and registered occupational therapist. Recruitment was conducted through a rigorous screening process to ensure participants met the following eligibility requirements: sufficient tactile sensitivity of the paretic arm; ability to understand written and verbal instructions; ability to provide verbal feedback; and sufficient functional movement to complete unimanual tasks using the paretic arm. Tactile sensitivity of the paretic arm was evaluated by a monofilament test; the degree of motor impairment was evaluated using the Functional Upper Extremity Disability Screening ${ }^{1}$ (FUEDS), which is a free online screening test; basic verbal communication was confirmed through participants' ability to express a sentence of three words or more; and cognitive skills were assessed through participants'

\footnotetext{
1 http://neurorehabconnection.com/index.php/free-screenings/upper-extremity-tool
}

ability to complete two-step instructions. All participants passed these tests with FUEDS scores of moderate (three participants) or minimal (two participants) motor impairment.

Apparatus: The firmware of the implementation was altered to accommodate the pilot study. The wristband on the paretic arm periodically delivered a vibration (cue) to convey a reminder to use the paretic arm. The cue was delivered every 30 seconds during 5 minute tasks. A fixed, rather than adaptive, cueing schedule was chosen to first test user tolerance and acceptance to vibrotactile cues while performing functional tasks. The tasks involved six activities, depicted in Fig. 2, commonly used by occupational therapists as part of stroke therapy procedures. During each task, both wristbands wirelessly transmitted acceleration data to a desktop computer for storage and offline analysis.

Procedure: Subjects first put on the wristbands with assistance from the experimenter. Subjects sat at a table adjusted so that its height was just above their lap. The study consisted of two conditions: completing tasks without vibrotactile cueing (condition $A$ ) or completing tasks with vibrotactile cueing (condition $B$ ). Each subject participated in both conditions (within-subject design) where condition order (AB or BA) was counterbalanced across subjects to reduce order effects with a 10-minute break between conditions. For each subject, each condition consisted of three randomly selected 5-minute tasks from Fig. 2. Subjects were instructed to use only their paretic arm to complete each task. As previously discussed, even with therapist recommendations and guidance, chronic stroke survivors often rely on and compensate with their unimpaired arm when difficulties are met using their paretic arm due to learned non-use. Only during condition $B$ were subjects told that they would feel vibrations, and to interpret these as reminders to use only their paretic arm.

Results: A post-experiment questionnaire was given to each participant to collect feedback on usability and tolerance of vibrotactile cueing and the overall system design. The mean subject responses to the Likert-scale questions are shown in Table I. The use ratio of the paretic arm and unimpaired arm were calculated (averaged across subjects and tasks) for both conditions. The paretic arm was used for $43.9 \%$ (SD: 28.1) and $35.1 \%$ (SD: 34.1 ) of the time during the tasks of condition $A$ and condition $B$, respectively. A paired $t$ test revealed no significant difference, $t(4)=1.31, p=0.26$, two-tailed. The unimpaired arm was used for $49.7 \%$ (SD: 39.1) and $49.0 \%$ (SD: 30.3 ) of the time during the tasks of condition $A$ and condition $B$, respectively. A paired $t$ test revealed no significant difference, $t(4)=0.085, p=0.937$, two-tailed.
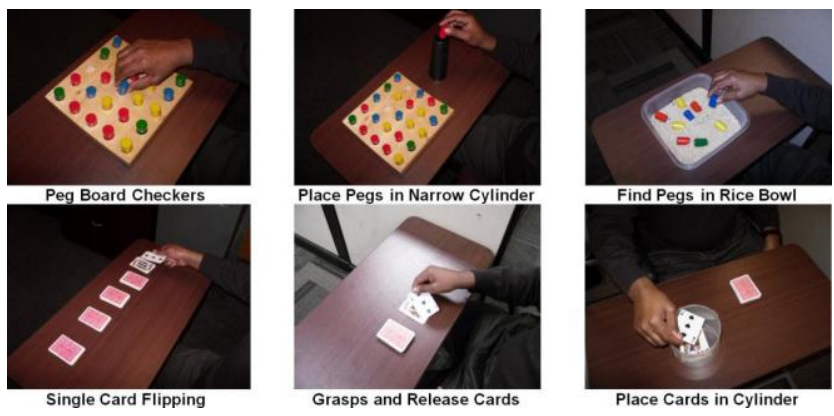

Figure 2. Six repetitive tasks used in pilot study. 
TABLE I. MEAN RESPONSES TO POST-EXPERIMENT QUESTIONS

\begin{tabular}{|c|c|c|c|}
\hline \multirow{2}{*}{ Q\# } & \multicolumn{3}{|c|}{ Likert-Scale Questions: 1 (low/negative) to 5 (high/positive) } \\
\hline & Usability Questions & Mean & $S D$ \\
\hline $1-1$ & $\begin{array}{l}\text { How easy was it to put on the wristband with } \\
\text { assistance? }\end{array}$ & 3.6 & 0.89 \\
\hline $1-2$ & $\begin{array}{l}\text { How easy was it to take off the wristband } \\
\text { with assistance? }\end{array}$ & 4.6 & 0.89 \\
\hline $1-3$ & $\begin{array}{l}\text { How easy was it to move your affected arm, } \\
\text { hand or fingers while wearing the wristband } \\
\text { compared to when you are moving without } \\
\text { wearing the wristband? }\end{array}$ & 4.8 & 0.45 \\
\hline $1-4$ & $\begin{array}{l}\text { How easy was it to grasp/hold objects with } \\
\text { your affected limb while wearing the } \\
\text { wristband compared to without wearing it? }\end{array}$ & 5.0 & 0 \\
\hline $1-5$ & How comfortable were the wristbands? & 4.6 & 0.55 \\
\hline \multirow[t]{2}{*}{$1-6$} & How lightweight were the wristbands? & 4.8 & 0.45 \\
\hline & Vibrotactile Design Questions & Mean & $S D$ \\
\hline $2-1$ & $\begin{array}{l}\text { How easy was it to learn that the vibrations } \\
\text { represented reminders to move only your } \\
\text { affected arm? }\end{array}$ & 4.6 & 0.55 \\
\hline $2-2$ & $\begin{array}{l}\text { How intuitive were the vibrations for remind- } \\
\text { ing you to move only your affected arm? }\end{array}$ & 4.2 & 0.84 \\
\hline $2-3$ & $\begin{array}{l}\text { How easy was it to recognize/notice the } \\
\text { vibrations during your session? }\end{array}$ & 4.4 & 0.89 \\
\hline $2-4$ & Do you feel that you missed any reminders? & 4.4 & 1.34 \\
\hline $2-5$ & Were the vibrations annoying? & 5.0 & 0 \\
\hline \multirow[t]{2}{*}{$2-6$} & Did the vibrations sometimes startle you? & 4.8 & 0.45 \\
\hline & Rehabilitation Questions & Mean & $S D$ \\
\hline $3-1$ & $\begin{array}{l}\text { How successful do you feel the wristband } \\
\text { performed at directing your attention to your } \\
\text { affected limb to overcome neglect when it } \\
\text { did not deliver vibrations versus when it did } \\
\text { deliver vibrations? a) Without vibrations; } \\
\text { b) With vibrations }\end{array}$ & $\begin{array}{l}\text { a) } 2.2 \\
\text { b) } 4.2\end{array}$ & a) 1.6 \\
\hline $3-2$ & $\begin{array}{l}\text { How successful do you feel the wristband } \\
\text { was at motivating you to use your affected } \\
\text { limb when it did not deliver vibrations versus } \\
\text { when it did deliver vibrations? a) Without } \\
\text { vibrations; b) With vibrations }\end{array}$ & $\begin{array}{l}\text { a) } 3.0 \\
\text { b) } 4.6\end{array}$ & a) 1.6 \\
\hline 3-3 & $\begin{array}{l}\text { How likely would you wear the vibrating } \\
\text { wristband at home for home-based rehab? }\end{array}$ & 4.2 & 1.79 \\
\hline $3-4$ & $\begin{array}{l}\text { How likely would you wear the vibrating } \\
\text { wristband in public for rehab any time/place? }\end{array}$ & 4.6 & 0.55 \\
\hline
\end{tabular}

Discussion: Subjects did not find the wristbands to be obtrusive nor hinder movement $(Q 1-3, Q 1-4)$. Subjects found the wristbands to be very comfortable ( $Q 1-5)$, lightweight $(Q 1$ $6)$ and easy to take off $(Q 1-2)$ with assistance. Subjects felt that the wristbands could have been easier to put on even with assistance (Q1-1); we are currently exploring easier donning methods. The vibrotactile cues were found to be very easy to learn $(Q 2-1)$, intuitive for their purpose $(Q 2-2)$, easy to recognize $(Q 2-3)$ and hard to miss $(Q 2-4)$. Subjects did not find the vibrations to be annoying (Q2-5) nor startling (Q2-6). Responses were positive and enthusiastic regarding the use of the proposed wearable computer outside of therapy sessions (Q3-3, Q3-4). Overall, subjects felt that cueing was successful at directing their attention and improving motivation to use their paretic arm (Q3-1,Q3-2). While this result is not reflected by objective data in which no difference was found in activity levels between conditions for either arm, this finding was expected. The clinical environment created a highly motivating setting for participants who respected the instruction to "use only your affected arm to complete each task" which was given in both conditions to ensure consistency in that the only difference between conditions was cueing. To better evaluate the effectiveness of cueing for overcoming learned non-use, an at-home user study over a few weeks involving activities of daily living is being planned. Even so, the presented pilot study provides promising results regarding tolerance and acceptance of vibrotactile cueing using wearable computers; and provides a starting point for bringing this technology into users' home and daily life outside of therapy sessions in clinics.

\section{CONCLUSION AND FUTURE WORK}

This paper presented an approach for overcoming learned non-use for stroke survivors using ubiquitous cueing via wearable computers. A pilot user study revealed user tolerance and acceptance of wearable computer-based vibrotactile cueing, and enthusiasm for use outside the clinic. As part of future work, an at-home user study is being planned to evaluate the effectiveness of vibrotactile cueing delivered based on adaptive performance goals. Effectiveness will be assessed in terms of functional outcomes resulting from enhanced awareness, and how these functional gains compare to CIMT.

\section{REFERENCES}

[1] V. L. Roger et al., "Heart disease and stroke statistics-2012 update: A report from the American Heart Association," Circulation, vol. 125, no. 1, pp. e2-e220, Jan. 2012.

[2] E. Taub, J. E. Crago, L. D. Burgio, T. E. Groomes, E. W. Cook, S. C. DeLuca, and N. E. Miller, "An operant approach to rehabilitation medicine: Overcoming learned nonuse by shaping," J Exp Anal Behav, vol. 61, no. 2, pp. 281-293, Mar. 1994.

[3] C. E. Lang et al., "Upper extremity use in people with hemiparesis in the first few weeks after stroke," Journal of Neurologic Physical Therapy, vol. 31, no. 2, pp. 55-63, Jun. 2007.

[4] W. S. Verplanck, "The operant conditioning of human motor behavior," Psychological Bulletin, vol. 53, no. 1, pp. 70-83, 1956.

[5] M. S. Cameirão, S. B. i Badia, E. Duarte, A. Frisoli, and P. F. M. J. Verschure, "The combined impact of virtual reality neurorehabilitation and its interfaces on upper extremity functional recovery in patients with chronic stroke," Stroke, vol. 43, no. 10, pp. 2720-2728, Oct. 2012.

[6] J. Lieberman and C. Breazeal, "TIKL: Development of a wearable vibrotactile feedback suit for improved human motor learning," IEEE Transactions on Robotics, vol. 23, no. 5, pp. 919-926, Oct. 2007.

[7] P. Kapur, M. Jensen, L. J. Buxbaum, S. A. Jax, and K. J. Kuchenbecker, "Spatially distributed tactile feedback for kinesthetic motion guidance," in IEEE Haptics Symposium, pp. 519-526, 2010.

[8] T. Markow et al., "Mobile Music Touch: Vibration stimulus in hand rehabilitation," in International Conference on Pervasive Computing Technologies for Healthcare (PervasiveHealth), pp. 1-8, 2010.

[9] P. Markopoulos, A. A. A. Timmermans, L. Beursgens, R. van Donselaar, and H. A. M. Seelen, 'Us'em: The user-centered design of a device for motivating stroke patients to use their impaired arm-hand in daily life activities," in Annual Conference of the IEEE Engineering in Medicine and Biology Society (EMBC), pp. 5182 -5187, 2011.

[10] G. Uswatte, C. Giuliani, C. Winstein, A. Zeringue, L. Hobbs, and S. L. Wolf, "Validity of accelerometry for monitoring real-world arm activity in patients with subacute stroke: Evidence from the extremity constraintinduced therapy evaluation trial," Archives of Physical Medicine and Rehabilitation, vol. 87, no. 10, pp. 1340-1345, Oct. 2006. 